\title{
Ultra-High Accurate Attitude Determination and Control of Microsatellite Formation Flight
}

\author{
N. S. Doszhan, G.E. Ibrayev and R.R. Pilpani
}

\begin{abstract}
Nowadays, technology and space science are developing rapidly, providing new possibilities for multi-segment missions to become a frequent practice. This relates to small satellites, which could be used to organize a formation, in order to solve complex scientific and applied problems. A formation could serve as distributed instruments for atmospheric sampling, construct a large distributed antenna platform, or make a large distributed aperture for imaging and other applications what only a big satellite can achieve. Small satellite's formation flying is low cost space project compared to one big satellite. However, to develop satellite formation a lot of problems should be solved depending on its mission.

One of the important tasks in small satellite formation is an accurate determination and control of position and attitude. This paper provides a mathematical modelling of a small satellite's formation motion, which is essential to realize a synthetic aperture telescope. Furthermore, the paper describes the principle of formation configuration and its design.
\end{abstract}

Keywords - geostationary orbit, formation flight, formation flying control, microsatellite.

\section{INTRODUCTION}

$I_{\text {of }}^{\mathrm{N}}$ N RECENT years, one of the most relevant tasks in the field of space monitoring especially for countries with large territories is observing of the Earth's surface in real time, which allows to monitor the rapid processes, such as, for example, the emergence and spread of steppe and forest fires. To achieve this goal, leading space agencies of the European Union, China and other countries are engaged in research and development of spacecrafts, which intended for remote sensing of the Earth from the geostationary orbit. Spacecrafts with a mass of several tons are not economically attractive, so recently an idea of launching a small spacecraft formation to a geostationary orbit appeared. For Kazakhstan which is

N.S. DOSZHAN, is with Department of Mechanics Kazakh National University named after al-Farabi, Almaty, Kazakhstan, (e-mail: nursultan.sagynaiuly@gmail.com).

G.E. IBRAYEV, is with Department of Mechanics Kazakh National University named after al-Farabi, Almaty, Kazakhstan, (e-mail: garyp.ybraev@gmail.com).

R.R. PILPANI, is with Department of Mechanics Kazakh National University named after al-Farabi, Almaty, Kazakhstan, (e-mail: pilpani.roland@gmail.com).

Manuscript received May 14, 2018; accepted October 20, 2018. DOI: $\underline{10.17694 / \text { bajece. } 475540}$ actively developing space activities in the field of remote sensing of the Earth and having a large territory, this idea is very promising When developing a small satellite formation, the most intricate problem is a formation motion control. Therefore, currently development of control system of satellite formation motion on geostationary is scientifically new and actual task.

As is known, remote sensing satellites in low Earth orbit cannot monitor with high frequency. The revisit interval with a period of less than one hour requires more than 200 satellites. Satellites formation on geostationary orbit has 24hour observability over a quarter of the Earth's surface, and data can be transmitted without time delay.

\section{THEORY}

It is planned to use the telescopes as payload of the small satellite formation. Nowadays the available place for the payload of the satellite limits the current capability of space telescopes. The telescope must fit both in mass and in size. This greatly limits the capability of the telescope since the distance observable is relayed to the baseline of the telescope.

In traditional telescopes, the baseline is the diameter of the primary mirror. Some effort has been made to generate deployable or segmented telescopes, but they are still fundamentally limited. Alternate option is launching separated satellites and organizing them into formation [1]. For a formation flying telescope, the effective baseline of the telescope is the distance between the satelltes. The light is collected from separated apertures, and then combined to form the image. The formation flight of the microsatellite is superior in cost, flexibility and robustness compared to a mission by one large satellite, and the future demand for synthetic aperture is remarkable [2].

There are four elements that are special to the formation flying problem: formation design, relative navigation, intersatellite communication, and formation control. These are described as follows.

\section{A. Formation design}

Formation design observed as the collective guidance problem for the desired location of each satellite as a function of time. It is dictated by the science needs of the mission. The dynamics of relative motion as applied to formation design continue to be a major research area. The problem is not only the specification of where the satellite needs to be as a function of time but also how to do this in the most fuelefficient manner. Concisely, formation design is the science of designing the desired relative motion of the vehicles to best meet science requirements without unreasonable fuel consumption. 


\section{B. Relative Navigation}

Relative navigation is the estimation of relative positions based on the measurements between adjacent satellites. Basically, the measurements are provided by sensors attached to the satellites. Accuracy class of the sensors depends on the purposes of mission.

\section{Communication}

The inter-satellite communication system is the data bus of the formation. More so than in other data buses, robustness and continuity are essential. The primary areas of development are mass, power and cost reduction, and integration of communications and ranging functions. This area includes hardware (transceivers or transponders), algorithms and network architectures, and software. Substantial work is still needed in developing requirements for communication bandwidth and time synchronization and transfer for precision formation control performance. Since the formation control laws are implemented through this system, a lack of integrity in the system will be a showstopper.

\section{Formation Control}

Formation control is responsible for rejecting disturbances, maintaining formation stability, and commanding the formation [3-4]. Specifically, this involves the application of forces and moments required to regulate and/or track desired formation geometry. Formation control includes the actuators, other components and algorithms, together with autonomy and higher-level command and control. The formation control function is heavily dependent on new technology. It is truly a system-level problem, depending critically on performance of the inter-satellite communications, the relative navigation, and the formation design. Formation control is the principal driver for concepts such as six degree of freedom satellite control and closed-loop orbit control.

\section{FORMATION FOR EARTH REMOTE SENSING IN REAL TIME}

Applying proper methods to solve the problem of each of the formation flying elements, the model of microsatellite formation with ultra-high accurate attitude determination and control could be developed.

Idea is to develop the formation flying of 4 microsatellites for Earth Remote sensing. This formation of small satellites should have very precise attitude control and determination. The main goal is to make an observation of the Earth (or part of Earth) near real time using low cost small satellite formation with ultra-high accuracy attitude control.

It is planned to use small satellite formation to generate one large synthetic aperture for scanning the Earth near real time. Using small satellite formation to the Low Earth Orbit for scanning the Earth near real time is impossible, because of high rotational speed of formation around the Earth, therefore image will be blurred. It is planned to put the formation to the geosynchronous orbit (GEO) which is very good point to observe the Earth in near real time.

First point is formation design. To solve problems of the mission, we need to use 4 microsatellites. They will consist of three small aperture telescopes and one combiner satellite to which the light from the three telescopes is relayed to be combined and detected. The distance between microsatellites will be $1 \mathrm{~m}$ (Fig.1).

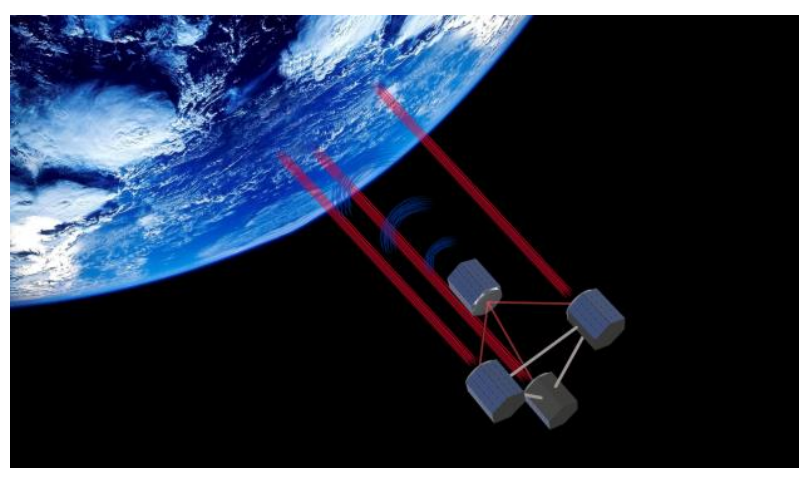

Fig.1. Satellite formation with aperture telescopes

It is planned to use the Fizeau type (Fig.2) synthetic aperture space telescope for each microsatellite to reach high resolution imaging [5].

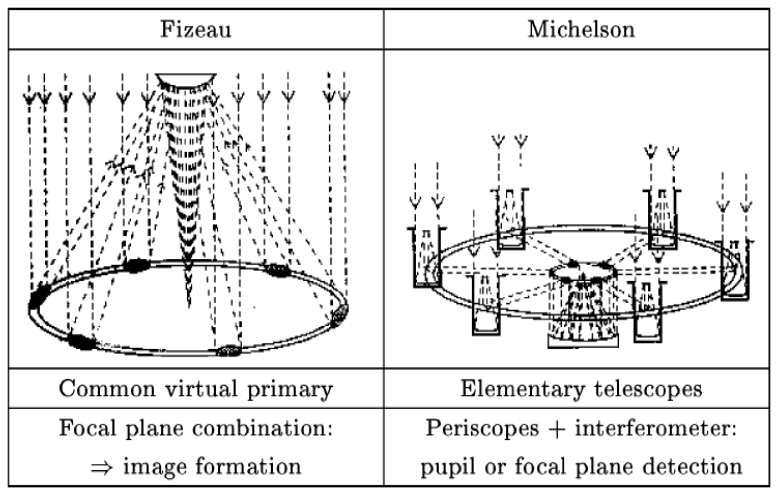

Fig.2. Imaging with multi-aperture optical telescopes and an application

A Fizeau synthetic aperture space telescope contains a set of mirrors forming a virtually common primary mirror, whose light is combined onto a common secondary mirror. The combination of the light beams coming from each piece of the primary mirror forms an image that is recorded in a common focal plane, in exactly the same way as for a monolithic telescope.

Secondly, to reach ultra-high accuracy of relative navigation between microsatellites it needs to be used very accurate sensor. Multiple sensor measurements can estimate the relative attitude and distance. In total, each microsatellite will involve 3-4 distance sensors [6-7].

Next stage is developing of formation control system (Fig.3). To change the attitude and position of satellites thrusters are necessary. Then again, within purposes of our mission the thrusters should be with high accuracy class [8]. As the accurate thruster we can use the $\mu$-PPT (micro pulsedplasma thruster) with very small impulse bits $(<25 \mu-\mathrm{N}-\mathrm{sec})$. 


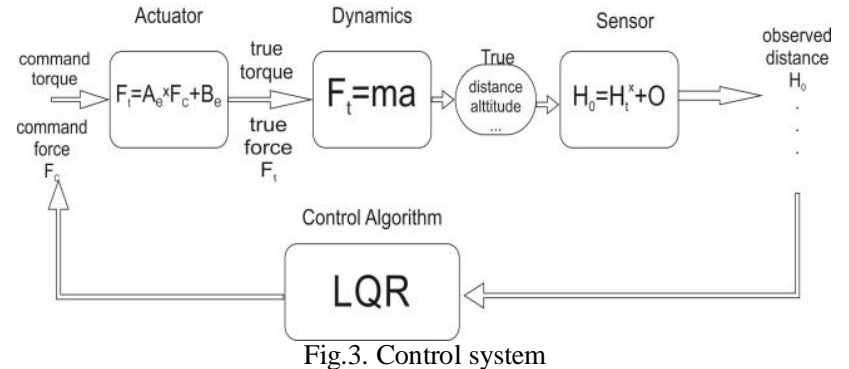

To success this mission there are some issues must be captured.

- For space science:

- Measurement requires high resolution but not so much amount of light is coming to the telescopes. Longer exposure time will be one way to solve it.

- Wave lengths which cannot be captured by ground telescope would be good target.

\section{- For remote sensing:}

- Bush fire detection would be good starting target, as it gathers much public attention.

- $100 \mathrm{~m} /$ pixel, $16 \mathrm{M}$ pixels can cover $400 \times 400 \mathrm{~km}$ (FOV) within one exposure time (say, $5 \mathrm{sec}$ )

- FOV is scanned (by some way) over places of interest

The area of 7,741,220 square kilometres can be scanned every 5.5 minutes. Mission itself should interest government to obtain the initial investment. GEO remote sensing outside of meteorological satellite field is rather new area. The bush fire detection satellite is only required during summer seasons. In other time, it can be used for space observation, etc. (wave length consideration is of course required). Even if the satellite cannot be inserted into GEO, many technological/mission demonstrations and tests are still possible in other orbits.

\section{MATHEMATICAL MODELLING OF FORMATION MOTION IN GEO}

The kinetic and potential energies for the formation from four satellite is the sum of each satellite's energy:

$$
\begin{aligned}
& E_{k}=E_{k_{1}}+E_{k_{2}}+E_{k_{3}}+E_{k_{4}} ; \\
& E_{p}=E_{p_{1}}+E_{p_{2}}+E_{p_{3}}+E_{p_{4}}
\end{aligned}
$$

where:

$E_{k}$ and $E_{p}$ - Kinetic and potential energies of the satellite formation.

The kinetic energy of a solid body by the König theorem [9]:

$$
\left.\begin{array}{l}
E_{k_{1}}=\frac{1}{2} m_{1} v_{1}^{2}+\frac{1}{2} J_{1} \omega_{1}^{2} ; \\
E_{k_{2}}=\frac{1}{2} m_{2} v_{2}^{2}+\frac{1}{2} J_{2} \omega_{2}^{2} \\
E_{k_{3}}=\frac{1}{2} m_{3} v_{3}^{2}+\frac{1}{2} J_{3} \omega_{3}^{2} ; \\
E_{k_{4}}=\frac{1}{2} m_{4} v_{4}^{2}+\frac{1}{2} J_{4} \omega_{4}^{2}
\end{array}\right\}
$$

where:

$m$ - mass of the satellite;

$v$ - velocity of the satellite;

$J$ - moment of inertia;

$\omega_{\text {- angular velocity. }}$

By the condition of the problem, the masses of three satellite with reflecting mirrors are equal.

Since all three satellites are at the same height, their speed is the same:

$$
v_{2}=v_{3}=v_{4}=v ; m_{2}=m_{3}=m_{4}=m
$$

Then, the total kinetic energy of the system:

$$
\begin{aligned}
& E_{k}=\frac{1}{2} m_{1} v_{1}^{2}+\frac{3}{2} m v^{2}+ \\
& +\frac{1}{2}\left[J_{1} \omega_{1}^{2}+J_{2} \omega_{2}^{2}+J_{3} \omega_{3}^{2}+J_{4} \omega_{4}^{2}\right]
\end{aligned}
$$

To calculate the potential energy, it is necessary to show all the forces acting on the satellite formation. The system is acted upon by the gravity forces of the Earth and the forces of mutual attraction of satellites (Fig.4).

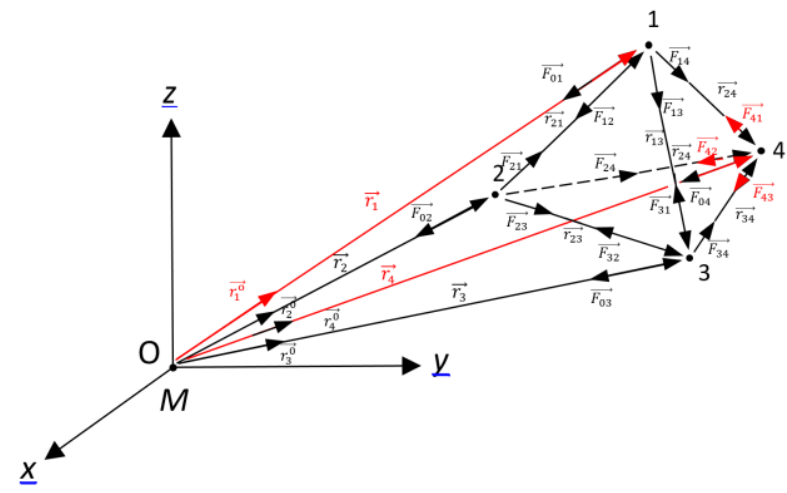

Fig.4. Gravity forces acting on the satellite formation Then the forces acting on the first satellite are equal:

$\overrightarrow{F_{01}}=-G \frac{M m}{r_{1}^{3}} \overrightarrow{r_{1}^{0}} \quad \overrightarrow{F_{12}}=-G \frac{m_{1} m}{r_{21}^{3}} \overrightarrow{r_{21}^{0}} \quad \overrightarrow{F_{13}}=G \frac{m_{1} m}{r_{13}^{3}} \overrightarrow{r_{13}^{0}}$

$\overrightarrow{F_{14}}=G \frac{m_{1} m}{r_{14}^{3}} \overrightarrow{r_{14}^{0}}$

where:

$\vec{F}$ - forces acting on the satellite formation;

$G$ - gravitational constant;

$M$ - mass of the Earth;

$m$ - masses of the satellites;

$r$ - distances between the centers of the masses.

By the same way, it is possible to calculate the forces acting on the other satellites:

$$
\begin{aligned}
& \overrightarrow{F_{02}}=-G \frac{M m}{r_{2}^{3}} \overrightarrow{r_{2}^{0}} \overrightarrow{F_{21}}=G \frac{m m_{1}}{r_{21}^{3}} \overrightarrow{r_{21}^{0}}, \\
& \overrightarrow{F_{23}}=G \frac{m^{2}}{r_{23}^{3}} \overrightarrow{r_{23}^{0}} \quad \overrightarrow{F_{24}}=G \frac{m^{2}}{r_{24}^{3}} \overrightarrow{r_{24}^{0}} ;
\end{aligned}
$$




$$
\begin{aligned}
& \overrightarrow{F_{03}}=-G \frac{M m}{r_{3}^{3}} \overrightarrow{r_{3}^{0}} \overrightarrow{F_{31}}=-G \frac{m m_{1}}{r_{31}^{3}} \overrightarrow{r_{31}^{0}}, \overrightarrow{F_{32}}=-G \frac{m^{2}}{r_{23}^{3}} \overrightarrow{r_{23}^{0}}, \\
& \overrightarrow{F_{34}}=G \frac{m^{2}}{r_{34}^{3}} \overrightarrow{r_{34}^{0}} ; \\
& \overrightarrow{F_{04}}=-G \frac{M m}{r_{4}^{3}} \overrightarrow{r_{4}^{0}} \overrightarrow{F_{41}}=-G \frac{m m_{1}}{r_{14}^{3}} \overrightarrow{r_{14}^{0}}, \\
& \overrightarrow{F_{42}}=-G \frac{m^{2}}{r_{24}^{3}} \overrightarrow{r_{24}^{0}} \overrightarrow{F_{43}}=-G \frac{m^{2}}{r_{34}^{3}} \overrightarrow{r_{34}^{0}}
\end{aligned}
$$

For a central force field, the potential energy is expressed as: $E_{p}=-\int \vec{F} d \vec{r}+$ Const

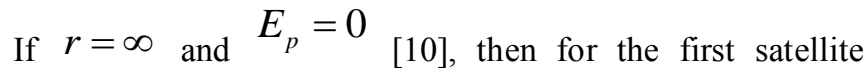
potential energy is:

$$
\begin{aligned}
& E_{p_{01}}=\int G \frac{M m_{1}}{r_{1}^{3}} \overrightarrow{r_{1}^{0}} d \overrightarrow{r_{1}^{0}}=G \frac{M m_{1}}{r_{1}^{3}} r_{1}^{2}=\frac{G M m_{1}}{\left|\overrightarrow{r_{2}}\right|} \quad E_{p_{12}}=\frac{G m_{1} m}{\left|\overrightarrow{r_{21}}\right|}, \\
& E_{p_{13}}=-\frac{G m_{1} m}{\left|\overrightarrow{r_{13}}\right|} \quad E_{p_{14}}=-\frac{G m_{1} m}{\left|\overrightarrow{r_{14}}\right|}
\end{aligned}
$$

By the same way, it is possible to calculate the potential energy of other satellites:

$$
\begin{aligned}
& E_{p_{02}}=\frac{G M m_{1}}{\left|\overrightarrow{r_{2}}\right|} \quad E_{p_{21}}=-\frac{G m m_{1}}{\left|\overrightarrow{r_{21}}\right|} ; \\
& E_{p_{23}}=-\frac{G m^{2}}{\left|\overrightarrow{r_{23}}\right|} \quad E_{p_{24}}=-\frac{G m^{2}}{\left|\overrightarrow{r_{24}}\right|} . \\
& E_{p_{03}}=\frac{G M m}{\left|\overrightarrow{r_{3}}\right|} \quad E_{p_{31}}=\frac{G m_{1} m}{\left|\overrightarrow{r_{31}}\right|} ; \\
& E_{p_{32}}=\frac{G m^{2}}{\left|\overrightarrow{r_{23}}\right|} ; \quad E_{p_{34}}=-\frac{G m^{2}}{\left|\overrightarrow{r_{34}}\right|} . \\
& E_{p_{04}}=\frac{G M m}{\left|\overrightarrow{r_{4}}\right|} \quad E_{p_{41}}=\frac{G m_{1} m}{\left|\overrightarrow{r_{41}}\right|} ; \\
& E_{p_{42}}=\frac{G m^{2}}{\left|\overrightarrow{r_{24}}\right|} \quad E_{p_{43}}=\frac{G m^{2}}{\left|\overrightarrow{r_{34}}\right|} .
\end{aligned}
$$

If assume that:

$$
\left|\overrightarrow{r_{23}}\right|=\left|\overrightarrow{r_{34}}\right|=\left|\overrightarrow{r_{24}}\right|=|\vec{r}|,\left|\overrightarrow{r_{13}}\right|=\left|\overrightarrow{r_{14}}\right|=\left|\overrightarrow{r_{12}}\right|=\left|\overrightarrow{r^{\prime}}\right|
$$

Then the potential energy of the system:

$$
E_{p}=G M\left[\frac{m_{1}}{\left|\overrightarrow{r_{1}}\right|}+m\left(\frac{1}{\left|\overrightarrow{r_{2}}\right|}+\frac{1}{\left|\vec{r}_{3}\right|}+\frac{1}{\left|\overrightarrow{r_{4}}\right|}\right)\right]
$$

To calculate the rotational motion relative to the center of mass, it is necessary to use the Koenig coordinate system relative to the center of inertia of the Earth. OXYZ is the coordinate system, where $\mathrm{O}$ is the center mass of the Earth. The $\mathrm{x}$-axis is directed along the Greenwich, the z-axis is directed along the rotation axis of the Earth, and the y-axis completes the coordinate system to the right (Fig.5).

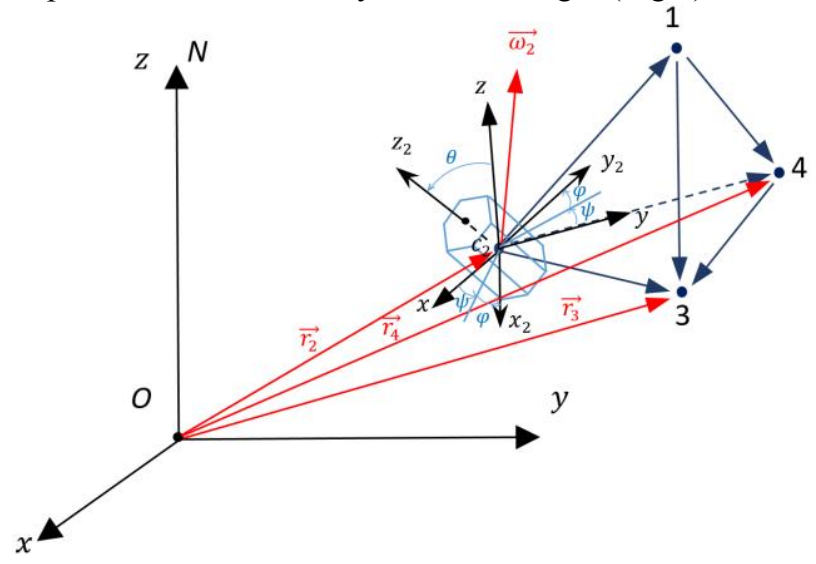

Fig.5. Koenig coordinate system relative to the center of inertia of the Earth.

$C X Y Z$ is Koenig coordinate system [11], $C_{2} X_{2} Y_{2} Z_{2}$ is principal axes of the satellite. The projection of the angular velocity vector $\overrightarrow{\omega_{2}}$ onto the movable axes:

$$
\left\{\begin{array}{c}
\omega_{2 x_{2}}=\dot{\psi}_{2} \sin \theta_{2} \sin \varphi_{2}+\dot{\theta}_{2} \cos \varphi_{2} \\
\omega_{2 y_{2}}=\dot{\psi}_{2} \sin \theta_{2} \cos \varphi_{2}-\dot{\theta}_{2} \sin \varphi_{2} \\
\omega_{2 z_{2}}=\dot{\varphi}_{2}+\dot{\psi}_{2} \cos \theta_{2}
\end{array}\right\}
$$

where: $\psi_{2}, \theta_{2}, \varphi_{2}$ - Euler angles for the second satellite. And the square of angular velocity module is:

$$
\omega_{2}^{2}=\dot{\psi}_{2}^{2}+\dot{\theta}_{2}^{2}+\dot{\varphi}_{2}^{2}+2 \dot{\varphi}_{2} \dot{\psi}_{2} \cos \theta_{2}
$$

By the same way, it is possible to calculate the square of angular velocity module for other satellites:

$$
\left.\begin{array}{l}
\omega_{1}^{2}=\dot{\psi}_{1}^{2}+\dot{\theta}_{1}^{2}+\dot{\varphi}_{1}^{2}+2 \dot{\varphi}_{1} \dot{\psi}_{1} \cos \theta_{1} \\
\omega_{3}^{2}=\dot{\psi}_{3}^{2}+\dot{\theta}_{3}^{2}+\dot{\varphi}_{3}^{2}+2 \dot{\varphi}_{3} \dot{\psi}_{3} \cos \theta_{3} \\
\omega_{4}^{2}=\dot{\psi}_{4}^{2}+\dot{\theta}_{4}^{2}+\dot{\varphi}_{4}^{2}+2 \dot{\varphi}_{4} \dot{\psi}_{4} \cos \theta_{4}
\end{array}\right\}
$$

Assuming that the generalized coordinates $q_{1}=x_{1}, q_{2}=y_{1}, q_{3}=z_{1}, q_{4}=\psi_{1}, q_{5}=\theta_{1}, q_{6}=\varphi_{1}$

and substituting Equations (17) and (18) into Equation (4), then using the Lagrange equations of the second kind, the following equations of motion of the system are obtained: 


$$
\left\{\begin{aligned}
\ddot{x}_{1}= & G M \frac{x_{1}}{r_{1}^{3}}, \dot{\psi}_{1}=\frac{\sin \varphi_{1}}{\sin \theta_{1}} p_{1}+\frac{\cos \varphi_{1}}{\sin \theta_{1}} q_{1} \\
\ddot{y}_{1}= & G M \frac{y_{1}}{r_{1}^{3}}, \dot{\theta}_{1}=p_{1} \cos \varphi_{1}-q_{1} \sin \varphi_{1} \\
\ddot{z}_{1}= & G M \frac{z_{1}}{r_{1}^{3}}, \\
\dot{\varphi}_{1}= & r_{1}-p_{1} \sin \varphi_{1} \operatorname{ctg} \theta_{1}-q_{1} \cos \varphi_{1} \operatorname{ctg} \theta_{1} \\
& J_{x} \dot{p}_{1}+\left(J_{z}-J_{y}\right) q_{1} r_{1}=M_{x_{1}} \\
& J_{y} \dot{q}_{1}+\left(J_{x}-J_{z}\right) p_{1} r_{1}=M_{y_{1}} \\
& J_{z} \dot{r}_{1}+\left(J_{y}-J_{x}\right) p_{1} q_{1}=M_{z_{1}}
\end{aligned}\right.
$$

\section{CONCLUSIONS}

Formation Flying is quickly revolutionizing the way the space community conducts autonomous science missions around the earth and in space. For Earth remote sensing it is possible to use other orbit than LEO, also it is very important to decrease the time and cost to develop new space technologies. This paper demonstrates how optimally we can achieve the almost impossible mission using modern technologies and science. One important goal of this work is possibility of microsatellite formations development in university level, which can totally replace the large spacecrafts in near future.

For the future, it is planned to study the impact of the solar radiation pressure to small satellite formation in GEO. In addition, it is planned to develop methods of nm-class sensing between microsatellites and control methods of this formation using multilevel actuators. Furthermore, the study of cooperative work of low thrust engine and proximity sensor of each satellite in formation will be implemented using MatLab

\section{ACKNOWLEDGMENT}

This work has been supported financially by the research project №AP05132939, for 2018-2020 of the al-Farabi Kazakh National University, which is gratefully acknowledged by the author.

\section{REFERENCES}

[1] Jesse Leitner. Formation Flying: The Future of Remote Sensing from Space. Proceedings of the International Symposium on Space Flight Dynamics, Munich, Oct., 2004, 11-15.

[2] Saptashi Bandyopadhyay, Rebecca Foust, Giri P. Subramanian, Soon-Jo Chung, and Fred Y. Hadaegh. Review of Formation Flying and Constellation Missions Using Nanosatellites. Journal of spacecrafts and Rockets, Vol. 53, No.3,1-12 (2016).

[3] D. C. Folta, L. K. Newman and T. Gardner. Foundations of Formation Flying for Mission to Planet Earth and New Millennium. AIAA, 1996, 656-666.
[4] Gokhan Inalhan, Franz D.Busse and Jonathan P.How. Precise formation flying control of multiple spacecraft using carrier-phase differential GPS. Proc. of AAS/AIAA Space Flight Mechanics Conference, Clearwater, FL, Paper No. AAS 00-109, January 23-26, 2000.

[5] Kun Liu, Yanfeng Qiao, Xiangyong Duan. Optical remote imaging using Fizeau synthetic aperture telescope. Proc. of Lasers \& Electro Optics \& The Pacific Rim Conference on Lasers and Electro-Optics, Shanghai China, August 31 - September 3, 2009.

[6] http://www.keyence.com/

[7] Journals William J Weber, Antonella Cavalleri, Rita Dolesi, Giorgio Fontana, Mauro Hueller, and Stefano Vitale. Position sensors for LISA drag-free control. Classical and Quantum Gravity, Vol.19, No.7, 17511752 (2002).

[8] Proceedings (Transactions, Book of abstracts, Proceedings on CD, etc.) John K. Ziemer and Stephen M. Merkowitz. Microthrust propulsion of the LISA mission. Proc. of 40th AIAA joint propulsion conference. Fort Lauderdale, Florida, July 12-14, 2004

[9] Yablonskiy A.A., Nikiforova V.M., Kurs teoreticheskoy mehaniki. $\mathrm{SPb} .:$ «Lan», 2001. $-504 \mathrm{p}$.

[10] Markeev A.P., Teoreticheskaya mehanika, M.: «CheRo», 1999. - 96 p.

[11] Beletskiy V.V., Dvizhenie sputnika otnositelno tsentra mass v gravitatsionnom pole, M.: 1975, $17 \mathrm{p}$.

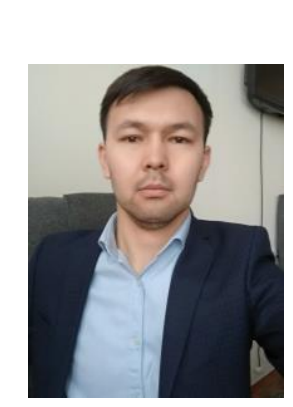

\section{BIOGR APHIES}

NURSULTAN DOSZHAN Republic of Kazakhstan, Aktobe city, in 1991. He received the B.S. degree in technical physics and M.S. degree in astronomy from the Kazakh National University named after Al-Farabi, Almaty city, B.S. in 2012 and M.S. in 2014. Since 2014, he has been researcher and senior teacher of the Department on Mechanics in al-Farabi Kazakh National University. $\mathrm{He}$ is the author of 10 articles, and the 4 international conferences. His research interests include small spacecraft design, spacecraft attitude determination and control, small spacecraft formation motion control

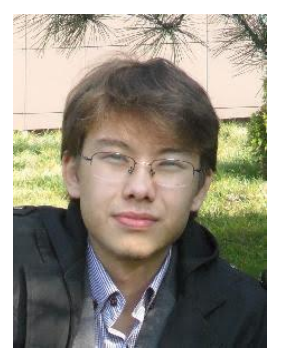

IBRAYEV GULAMA-GARIP ALISHER Republic of Kazakhstan, Uralsk city, in 1992. He received the B.S. degree in space technic and technologies and M.S. degree in space technic and technologies from the Kazakh National University named after Al-Farabi, Almaty city, B.S. in 2014 and M.S. in 2016. Since 2017, he has been PhD student, researcher and lecturer of the Department of Mechanics in al-Farabi Kazakh National University. He is the author of 1 book, 15 articles, and the 7 international conferences. His research interests include astrodynamics, solid mechanics, nonlinear oscillations and rotor dynamics

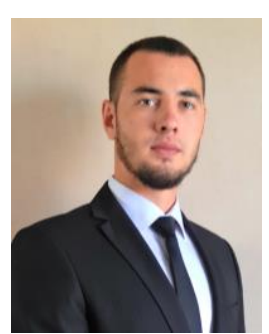

PILPANI ROLAND Almaty City, in 1997. He was enrolled in Kazakh National University named after al-Farabi in 2015, on the Faculty of Mechanics and Mathematics; specialty is "Space Engineering and Technologies".

Since 2017, he has been a Laboratory Assistant. His research interests include designing and application of small spacecrafts, control system of spacecraft, satellite formation flying control systems. 